\title{
Linagliptin Ameliorates Pulmonary Fibrosis in \\ Systemic Sclerosis Mouse Model via Inhibition of Endothelial to Mesenchymal Transition
}

\section{Biwei Pei}

The First Affiliated Hospital of Anhui Medical University

\section{$\mathrm{Na}$ Zhang}

The First Affiliated Hospital of Anhui Medical University

\section{Tingting Pang}

The First Affiliated Hospital of Anhui Medical University

Gengyun Sun ( $\nabla$ sungengy@126.com )

The First Affiliated Hospital of Anhui Medical university https://orcid.org/0000-0001-9886-6563

\section{Research Article}

Keywords: Linagliptin, Pulmonary fibrosis, Systemic sclerosis, Endothelial to mesenchymal transition, oxidative stress

Posted Date: September 14th, 2021

DOl: https://doi.org/10.21203/rs.3.rs-873241/v1

License: (9) (1) This work is licensed under a Creative Commons Attribution 4.0 International License. Read Full License

Version of Record: A version of this preprint was published at Molecular and Cellular Biochemistry on January 5th, 2022. See the published version at https://doi.org/10.1007/s11010-021-04349-1. 


\section{Abstract}

Systemic sclerosis (SSc) is a connective tissue disease that often causes pulmonary fibrosis. Dipeptidyl peptidase 4 (DPP4) inhibitor, has shown anti-fibrotic properties in various fibrotic diseases. However, only two studies have reported its anti-fibrosis effects in pulmonary fibrosis, and the mechanism is not completely clear. In the present study, we further investigated the protective effects of linagliptin, a highly specific DPP4 inhibitor, on pulmonary fibrosis in SSc mouse model and the potential mechanisms. The results showed that linagliptin ameliorated pulmonary fibrosis in SSc mouse model, as evidenced by improved pathological changes of lung and body weight loss induced by BLM. Linagliptin also reduced BLM-induced oxidative stress, inflammation in lung in vivo. We revealed that linagliptin attenuated BLMinduced endothelial to mesenchymal transition (EndMT) in vitro and in vivo. BLM-induced enhanced migration ability of endothelial cells was also alleviated by linagliptin. Moreover, we confirmed that the Akt/mammalian target of rapamycin (mTOR) pathway was involved in BLM-induced EndMT in vivo, which was suppressed by linagliptin. In summary, we further confirmed the therapeutic effects of linagliptin on pulmonary fibrosis in SSc mouse model, which is based on its inhibitory effects on EndMT, oxidative stress and inflammation.

\section{Introduction}

Systemic sclerosis (SSc), a rare autoimmune-mediated connective tissue disease often causes progressive pulmonary fobrosis [1], which causes a high mortality [2]. A previous study has reported that pulmonary fibrosis or interstitial lung disease is present in $80 \%$ of patients with SSc [3]. However, treatment for SSc-related pulmonary fibrosis is limited. Therefore, development of novel therapeutic approaches is an urgent need to improve the clinical benefit and quality of life of patients with SScrelated pulmonary fibrosis.

The pathogenesis of SSc-related pulmonary fibrosis is complicated and poor understanding. Myofibroblasts, an unique population of mesenchymal cells, play a pivotal role in the pathogenesis of fibrotic diseases. The cells display a marked profibrotic cellular phenotype including an increased production of fibrillar type I and type III collagen, initiation of the expression of a-smooth muscle actin (aSMA) and reduced expression of genes encoding extracellular matrix degradative enzymes [4]. Zeisberg EM et al reported that in cardiac fibrosis, a part of myofibroblasts were derived from endothelial cells via endothelial to mesenchymal transition (EndMT) [5], which is a transdifferentiation process first found in the embryonic developmental process. Subsequently, the process has been demonstrated to be involved in the progression of various fibrotic diseases $[6,7,8]$, as well as SSc-related pulmonary fibrosis [9]. Given the critical role of myofibroblasts in the pathogenesis of SSc, treatments targeting EndMT are promising therapies.

Dipeptidyl peptidase 4 (DPP4), also known as CD26, is a serine protease and a type II transmembrane glycoprotein expressed in various cell types including endothelial cells [10]. DPP4 inactivates incretin hormones such as glucagon-like peptides and glucose-dependent insulinotropic peptide. DPP4 inhibitor 
has been widely used for the treatment of type 2 diabetes because of its activity against DPP4 [11, 12]. DPP4 has also been found to be involved in fibrotic diseases. Previous investigations have demonstrated that the DPP4 expression and the number of DPP4-positive fibroblasts were increased in fibrotic skin of SSc and keloid patients and that DPP4-positive fibroblasts expressed higher levels of myofibroblast markers and collagen, indicating that DPP4 may be a marker of myofibroblasts [13, 14]. Moreover, two studies have reported the therapeutic effects of DPP4 inhibitor on pulmonary fibrosis by inactivating fibroblasts, regulating extracellular matrix and anti-inflammatory effects in mice induced by intratracheal administration of bleomycin (BLM) $[13,15]$. However, relevant research is still lacking. Therefore, further elucidation of the mechanisms of its antifibrotic activity will contribute to its application in the treatment of SSc-related pulmonary fibrosis.

This study aimed to investigate the effects of linagliptin, a highly specific DPP4 inhibitor, on pulmonary fibrosis in SSc mouse model and potential underlying mechanisms. The results further demonstrated the protective effects of linagliptin on pulmonary fibrosis, which may attribute to its inhibition of EndMT, oxidative stress and inflammation.

\section{Materials And Methods}

\section{Reagents}

BLM, linagliptin and rapamycin were purchased from Selleck (Houston, TX, USA). Primary antibodies against CD31, VE-Cadherin and a-smooth muscle actin (a-SMA) were purchased from Abcam (Cambridge, UK). Primary antibodies against Snail, Slug, Twist, Akt, phosphorylated Akt, mammalian target of rapamycin (mTOR) and phosphorylated mTOR were purchased from Cell Signaling Technology (Danvers, MA, USA). Primary antibodies against type I collagen were obtained from Wanleibio (Shenyang, China). Primary antibodies against $\beta$-actin were obtained from Proteintech (Wuhan, China).

\section{Animals}

6-8 weeks female C57BL/ 6 mice, weighing 20-22 g, were obtained from the Laboratory Animal Center of Anhui Medical University (Hefei, China) and housed in a SPF room with controlled temperature and humidity. All mice were supplied with a standard rodent diet and free water.The back of the mice was shaved, and then the mice were randomly divided into four groups: control, BLM, BLM + linagliptin and $\mathrm{BLM}+$ rapamycin. Rapamycin was used as a positive control for the involvement of the Akt/mTOR pathway in both the BLM-induced SSc mouse model [16] and the BLM-induced EndMT in human umbilical vein endothelial cells (HUVECs) [17]. The mice in the control group were subcutaneously injected with $0.1 \mathrm{~mL}$ of phosphate-buffered saline (PBS) with a 4.5-gauge needle. The mice in the other groups were injected with $0.1 \mathrm{~mL}$ of BLM solution $(1 \mathrm{mg} / \mathrm{mL})$ daily for 4 weeks. After the first week of injections, the mice in the control, BLM + linagliptin and BLM + rapamycin groups were orally administrated daily with PBS, linagliptin $(10 \mathrm{mg} / \mathrm{kg})$ and rapamycin $(1.5 \mathrm{mg} / \mathrm{kg})$, respectively. All mice were anesthetized and sacrificed on day 28. All experiments were approved by the Animal Care and Use Committee of Anhui Medical University (Hefei, China). 


\section{Measurement of hydroxyproline, superoxide dismutase (SOD) and 3,4-methylenedioxyamphetamine (MDA) in lung tissues}

Lung tissues were homogenized in PBS, centrifuged at $12000 \cdot \mathrm{g}$ for $10 \mathrm{~min}$, and then the supernatant was collected for further analysis. The hydroxyproline (A030-2-1, Jiancheng, Nanjing, China), SOD (A0013-1, Jiancheng) and MDA (A003-1-1, Jiancheng) contents in the lungs were determined following the manufacturer's instructions.

\section{Measurement of tumor necrosis factor (TNF)- $a$ and interleukin (IL)-6 in bronchoalveolar lavage fluid (BALF)}

BALF was harvested and centrifuged at $1000 \cdot \mathrm{g}$ for $10 \mathrm{~min}$. Then, the supernatant was collected and stored at $-80^{\circ} \mathrm{C}$ for further analysis. The levels of TNF-a and IL- 6 were determined using TNF-a enzymelinked immunosorbent assay (ELISA) kits (PI326, Beyotime Biotechnology, Shanghai, China) and IL-6 ELISA kits (PT512, Beyotime Biotechnology) in accordance with the manufacturer's instructions.

\section{Cell culture}

HUVECs were purchased from Sciencell Research Laboratories (San Diego, CA, USA) and cultured in endothelial culture medium supplemented with $5 \%$ fetal bovine serum, $1 \%$ endothelial cell growth supplement and $1 \%$ penicillin-streptomycin solution. The cells were maintained in an incubator containing $5 \% \mathrm{CO} 2$ at $37^{\circ} \mathrm{C}$. Cells were used in passages $2-7$. The cells were seeded in 6-well plates and treated as follows: control, BLM $(2 \mu \mathrm{g} / \mathrm{mL}), \mathrm{BLM}(2 \mu \mathrm{g} / \mathrm{mL})+$ linagliptin $(100 \mathrm{nM})$ or BLM $(2 \mu \mathrm{g} / \mathrm{mL})+$ rapamycin (200 $\mathrm{nM})$. After $48 \mathrm{~h}$ of treatment, the cells were harvested for further analysis. An inverted microscope (CKX53, Olympus, Tokyo, Japan) was used to observe and photograph changes in cell morphology.

\section{Scratch assay and migration assay}

For scratch assay, cells were seeded in 6-well plates. A scratch was evenly created by $200 \mu$ l pipette tip crossing the surface of the cell monolayer and detached cells were washed off. Then cells were incubated with serum-free medium containing indicated drugs. Images were taken at different times. For migration assay, cells were suspended with serum-free medium and then 200 ul cell suspension with indicated drugs were plated in Matrigel (BD Biosciences) pre-coated transwell chamber with 8-um pore. The chamber was then placed into 12-well plate containing 800 ul culture medium supplemented with 5\% fetal bovine serum and cultured. After $24 \mathrm{~h}$ migration, the migrated cells were fixed with $4 \%$ paraformaldehyde, stained with crystal violet and photographed.

\section{Histological analysis and immunohistochemistry}

Lungs were fixed in $4 \%$ paraformaldehyde, embedded in paraffin, cut into $4-\mu m$-thick slices, and then subjected to Masson's trichrome staining and hematoxylin and eosin (H\&E) staining. For 
immunohistochemical staining, after the slices were dewaxed and rehydrated, they were incubated with citrate buffer for antigen retrieval. Endogenous peroxidase activity and non-specific binding were blocked with $3 \%$ hydrogen peroxide and $5 \%$ goat serum, respectively. Then, the slices were incubated with primary antibodies against CD31 (1:100), a-SMA (1:200) or Slug (1:200) at $4^{\circ} \mathrm{C}$ overnight, followed by secondary antibodies at $37^{\circ} \mathrm{C}$ for $1 \mathrm{~h}$. The stained slices were visualized with diaminobenzidine, counterstained with hematoxylin and observed by bright field imaging using a fluorescence microscope (DM6 B; Leica, Germany).

\section{Immunofluorescence analysis}

For lung tissues immunofluorescence, after deparaffinization, antigen retrieval and blockade, the slices were incubated with CD31 (1:100) and a-SMA (1:200) antibodies at $4^{\circ} \mathrm{C}$ overnight, followed by incubation with Alexa 488 Goat Anti-rabbit IgG and Alexa 647 Goat Anti-mouse IgG at room temperature for $1 \mathrm{~h}$. For in vitro immunofluorescence, HUVECs were seeded on glass coverslips in 24-well culture plates and treated as indicated. The cells were washed and fixed with $4 \%$ paraformaldehyde, permeabilized with $0.3 \%$ Triton $X-100$, and then blocked with $5 \%$ bovine serum albumin at room temperature. The fixed cells were incubated with primary antibodies against VE-Cadherin (1:200) and a-SMA (1:200), or Slug (1:200) at $4^{\circ} \mathrm{C}$ overnight, followed by incubation with the appropriate secondary antibodies at room temperature for 1 h. 4',6-diamidino-2-phenylindole (DAPI) was used to stain the nuclei. All samples were visualized and photographed using a laser scanning confocal microscope (LSM 880; Carl Zeiss, Oberkochen, Germany) or a fluorescence microscope (DM6 B; Leica).

\section{Real-time quantitative PCR}

Total RNA of HUVECs was isolated using Trizol (Invitrogen, Carlsbad, CA, USA), and then reverse transcribed to cDNA using a TaKaRa PrimeScript ${ }^{\text {TM }}$ RT Master Mix kit (Shiga, Japan). TaKaRa TB GREEN Premix Ex Taq (Kyoto, Japan) and Applied Biosystems QuantStudio6 Flex real-time PCR system (Thermo Fisher Scientific, Waltham, MA, USA) were used for real-time quantitative PCR (RT-qPCR). $\beta$-actin was used as an internal control. The primers were designed and synthesized by General Biosystems (Chuzhou, China) and the sequences are shown in Table 1. Gene expression levels were normalized to $\beta$ actin, and relative fold changes in mRNA expression were determined using the $2-\Delta \Delta \mathrm{Ct}$ method. 
Table 1

Primer sequences for RT-qPCR

\begin{tabular}{|ll|}
\hline Primer & Sequence(5'-3') \\
\hline F-CD31 & AACGGAAGGCTCCCTTGATG \\
\hline F-CD31 & TAAGAACCGGCAGCTTAGCC \\
\hline R-VE-Cadherin & GGCTCATGTATCGGAGGTCG \\
\hline F-a-SMA & TATCCCCGGGACTAAGACGG \\
\hline R-a-SMA & CACCATCACCCCCTGATGTC \\
\hline F-COL1A1 & TCCCAGGGTGGCTTCTGATA \\
\hline R-COL1A1 & GAGTCGGGGACACTTACAGC \\
\hline F-Snail & CGAGTGGTTCTTCTGCGCTA \\
\hline R-Snail & CTGCTGGAAGGTAAACTCTGGA \\
\hline F-Slug & CTGGGCTGGCCAAACACAA \\
\hline R-Slug & GCTCACATATTCCTTGTCACAGAAC \\
\hline F-Twist & GCCGGAGACCTAGATGTCATT \\
\hline R-Twist & TTTTAAAAGTGCGCCCCACG \\
\hline F- $\beta$-actin & CTTCCAGCCTTCCTTCCTGG \\
\hline R- $\beta$-actin & CTTCTGCATCCTGTCGGCAA \\
\hline
\end{tabular}

\section{Western blotting}

Total protein of tissues and cell lysates were extracted using ice-cold RIPA lysis buffer supplemented with $1 \mathrm{mM}$ phenylmethylsulfonyl fluoride. Then, the lysates were subjected to ultrasonication. The protein concentrations were measured using a BCA protein assay kit. Equal amounts of protein were loaded on sodium dodecyl sulfate-polyacrylamide gels, electrophoresed and transferred to polyvinylidene fluoride membranes, which were blocked and incubated with primary antibodies overnight at $4^{\circ} \mathrm{C}$. Subsequently, the membranes were incubated with secondary antibodies for $2 \mathrm{~h}$ at room temperature, followed by detection with ECL reagents. The primary antibodies and dilutions used were as follows: anti-CD31 (1:500), anti-VE-Cadherin (1:1000), anti-a-SMA (1:500), anti-Collagen I (1:500), anti-Snail (1:1000), antiSlug (1:1000), anti-Twist (1:1000), anti-Akt (1:1000), anti-P-Akt (1:1000), anti-mTOR (1:1000) and anti-PmTOR (1:1000). Anti- $\beta$-actin (1:10000) was used as an internal control. Selected blots were quantified using Image $\mathrm{J}$.

\section{Statistical analysis}


The data are presented as mean \pm standard deviation. One-way analysis of variance was used to analyze statistical differences between groups, followed by the post hoc least significant difference test. P $<0.05$ was considered statistically significant. All analyses were conducted using SPSS version 22.0. GraphPad Prism 6 software was used to generate the graphs.

\section{Results}

\section{Linagliptin ameliorated pulmonary fibrosis in SSc mouse model}

All the mice were sacrificed on day 28 and lungs were harvested for further analysis. The BLM-treated mice showed a significant body weight loss compared with the control mice, whereas the linagliptin or rapamycin interventions reversed this trend (Fig. 1a). H\&E staining of lung sections showed that subcutaneous injection of BLM resulted in destruction of normal lung structures and marked thickening of alveolar septa. Masson's trichrome staining showed a significant increase in collagen deposition in the lungs of BLM-treated mice. However, both linagliptin and rapamycin significantly alleviated these pathological changes and reduced collagen production (Fig. 1d, e). Consistently, linagliptin or rapamycin treatments reduced the BLM-induced increased levels of hydroxyproline, a marker of collagen protein in lungs (Fig. 1c). In the Ashcroft Fibrosis Scale, the linagliptin and rapamycin groups had lower scores than those of the BLM group (Fig. 1b).

\section{Linagliptin mitigated BLM-induced inflammation and oxidative stress}

To evaluate the effect of linagliptin on BLM-induced pulmonary inflammatory responses, the levels of TNF- $a$ and IL- 6 in the BALF were determined. We found that BLM administration induced increased levels of TNF- $a$ and IL-6 in the BALF, and that these levels were reduced by linagliptin or rapamycin treatments (Fig. 2a, b). Considering the important role of oxidative stress in the induction of pulmonary fibrosis in SSc [18], we measured the MDA content and SOD activity in the lungs, both of which are accepted oxidative stress markers [19]. As shown in Fig. 2c and d, the MDA content was increased and the activity of SOD was significantly decreased in the lungs of BLM-treated mice. In contrast, linagliptin or rapamycin co-treatment remarkably suppressed the BLM-induced alterations in SOD and MDA.

\section{Linagliptin attenuated BLM-induced EndMT in vitro}

HUVECs are commonly used in in vitro studies related to endothelial cell function, including EndMT in pulmonary fibrosis $[20,21]$. In the present study, BLM treatment for $48 \mathrm{~h}$ induced HUVECs to transform from a cobblestone-like shape to an elongated, spindle shape, but this was attenuated by linagliptin or rapamycin treatments (Fig. 3a). At the molecular level, western blotting results revealed that BLM induced decreased protein levels of endothelial markers (CD31 and VE-Cadherin) and increased levels of mesenchymal marker (a-SMA and Collagen I) in HUVECs. However, these alterations were suppressed by linagliptin or rapamycin treatments (Fig. 3b). Immunofluorescence analysis further confirmed these results (Fig. $3 g$ ). In accordance with the western blotting and immunofluorescence analysis, RT-qPCR analysis showed that BLM upregulated the mRNA expression of CD31 and VE-Cadherin and 
downregulated the mRNA expression of a-SMA and Collagen I, and that these effects were inhibited by linagliptin or rapamycin treatment (Fig. 3c, d, e, f).

\section{Lingaliptin inhibited BLM-induced enhanced migration ability}

HUVECs undergoing EndMT lose cell-cell adhesion and acquired an enhanced migration ability [17]. Scratch and migration assay were performed to evaluated the effects of linagliptin on migration ability of HUVECs. The results showed that BLM treatment significantly enhanced the migration ability of HUVECs, which was attenuated by linagliptin or rapamycin treatment (Fig. 4a,b).

\section{Linagliptin attenuated BLM-induced EndMT in vivo}

CD31/a-SMA double-labeling immunofluorescence was used to evaluate EndMT in vivo. Co-localization of CD31/a-SMA in the endothelium indicates the intermediate stages of EndMT. The BLM group had more double-positive cells compared with the control group; however, this elevated percentage of doublepositive cells in the BLM-treated group was reversed in the linagliptin or rapamycin-treated mice (Fig. 5a). Immunohistochemistry analysis showed that the expression of CD31, which is mainly localized at the intima of the capillary vessels, was decreased in the BLM group compared with that in the control group, whereas elevated levels of a-SMA-positive cells were observed in the BLM group compared with the control group (Fig. 5b, c). These expression changes in EndMT-related markers were suppressed by linagliptin or rapamycin treatment.

\section{Linagliptin inhibited EndMT-related transcription factors in vitro and in vivo}

Snail, Slug and Twist are key transcription factors in EndMT induction. In vitro experiments revealed that the protein and mRNA levels of these factors were significantly elevated in the BLM group compared with those in the control group, however linagliptin or rapamycin treatment suppressed these BLM effects (Fig. 6a, b, c, d). Immunofluorescence analysis showed that linagliptin or rapamycin attenuated the increased amount of Slug located in the nucleus induced by BLM (Fig. 6e).

Next, we investigated the effect of linagliptin on EndMT-related transcription factors in vivo. Consistently, all the above mentioned transcription factors' protein levels were increased in the BLM group, compared with the control group. However, linagliptin or rapamycin treatment significantly reversed the BLMinduced increased protein levels of these factors (Fig. 7a). Furthermore, immunohistochemistry analysis showed an increased number of Slug-positive cells in the vessel inner wall in the BLM group compared with the control group, and this upregulation was significantly reduced in the linagliptin and rapamycin groups (Fig. 7b).

\section{Linagliptin inhibited the Akt/mTOR pathway in vivo}

Activation of the Akt/mTOR pathway is an important inducer of EndMT [17]. It has also been demonstrated that inhibition of this pathway reversed BLM-induced EndMT [16, 22]. Hence, we explored the effect of linagliptin on activation of the Akt/mTOR pathway. Western blot analysis revealed that BLM 
significantly upregulated the phosphorylation level of Akt and mTOR in SSc mice, and that this effect was reversed by linagliptin or rapamycin treatment (Fig. 8). Taken together, the results indicated that the Akt/mTOR pathway is involved in the inhibition effect of linagliptin on BLM-induced EndMT.

\section{Discussion}

DPP4 inhibitors have attracted much attention since their anti-fibrosis effects have been reported. Here, we further confirmed the protective effects of linagliptin on pulmonary fibrosis in SSc mouse model and revealed that suppression of EndMT, oxidative stress, inflammation are important mechanisms underlying the effects.

Intratracheal administration of BLM cause severe acute lung injury followed by pulmonary fibrosis and inflammation in mice [23], which is the most commonly used animal model for studying idiopathic pulmonary fibrosis. Subcutaneous injection of BLM also induce pulmonary fibrosis with a chronic lung injury and more insidiously progressive course, which was more similar to pulmonary fibrosis in SSc patients, including patchy interstitial fibrosis and mononuclear cell infiltration. Furthermore, Yoshizaki A et al have reported that SSc-related antibodies such as anti-Scl-70, anti-U1RNP and anti-CENP B were positive in the sera of mice treated with subcutaneous injection of BLM [23]. Therefore, subcutaneous injection of BLM may better mimic SSc-related fibrosis. And it has been commonly used to study skin and pulmonary fibrosis associated with SSc $[23,24,25,26,27]$. So we treated mice with BLM subcutaneously to induce pulmonary fibrosis in the present study. The results showed that subcutaneous BLM administration caused loss of normal lung structure, thickened alveolar walls and increased collagen fiber content, accompanied by significant weight loss. These BLM-induced effects were alleviated by linagliptin treatment. Lung hydroxyproline content was also remarkably reduced by linagliptin treatment. The results further validated the protective effects of linagliptin on pulmonary fibrosis, which was consistence with previous studies $[13,15]$.

Myofibroblasts are the main orchestrators of fibrosis by synthesizing and releasing a large amount of extracellular matrix proteins, leading to structure destruction and function loss of normal tissues and organs [28]. Myofibroblasts emerge from several sources, including expansion and activation of quiescent resident tissue fibroblasts [29], migration and tissue accumulation of bone marrow-derived circulating CD34+ fibrocytes [30] and epithelial cells or perivascular cells (pericytes) that have undergone a phenotypic transition into mesenchymal cells [31]. EndMT is another important source of myofibroblasts newly discovered in recent years [8]. In this complex biological process, endothelial cells lose their endothelial characteristics and gain mesenchymal cell characteristics, including a spindleshaped cell morphology, loss of polarity and intercellular junctions, and enhanced migration ability [32]. EndMT also results in the initiation of expression of mesenchymal cell specific proteins, such as a-SMA, vimentin, $\mathrm{N}$-cadherin. Subsequently, the involvement of EndMT in SSc related lung diseases was also confirmed [9]. Especially in SSc, the occurrence of EndMT not only makes endothelial cells an additional source of myofibroblasts, but also indirectly leads to a defective angiogenesis, an important pathogenesis in SSc [33]. Therefore, EndMT has become an interesting target for SSc treatment. 
Vildagliptin, a DPP4 inhibitor, have been reported to improve lipopolysaccharide-induced lung injury by inhibiting EndMT [34]. Therefore, EndMT is also likely to be a potential mechanism by which DDP4i ameliorates BLM-induced pulmonary fibrosis.

In the present study, we treated HUVECs with BLM for 48h and induced EndMT in vitro, similarly to a previous study [17]. Our results showed that linagliptin co-treatment attenuated BLM-induced EndMT in vitro. BLM-induced enhanced migration of cells was also alleviated. BLM can also induce EndMT in vivo [35]. In the study, the lungs of BLM-treated mice developed EndMT, as evidenced by the increased number of co-localized CD31/a-SMA cells observed in the lungs of the BLM group, compared with the control group. As expected, the BLM-induced EndMT was inhibited by linagliptin in vivo.

Transcription factors including Snail, Slug and Twist are key mediators of EndMT. It has been confirmed that siRNA knockdown of Snail blocked transforming growth factor (TGF)- $\beta$-induced EndMT [36], Slug silencing reversed BLM-induced EndMT [17] and Twist overexpression induced EndMT in cultured human pulmonary arterial endothelial cells [37]. Thus, we examined the effect of linagliptin on the expression of these transcription factors. BLM upregulated the expression of Snail, Slug and Twist in vitro and in vivo, while linagliptin treatment abrogated this effect. Therefore, inhibition of EndMT is another important mechanism by which linagliptin improves pulmonary fibrosis in SSc mouse model.

The mechanisms underlying the effect of linagliptin on EndMT have not been fully elucidated. Shi et al. have reported that the interaction of DPP4 with integrin $\beta 1$ promotes the TGF- $\beta$-induced TGF- $\beta$ receptor heterodimer formation, initiates the Smad-dependent pathway and ultimately leads to EndMT. Linagliptin inhibited the EndMT process by suppressing the expression of DPP4 and integrin $\beta 1$ [38]. However, the exact mechanisms of the protective effects of linagliptin on BLM-induced EndMT need to be further studied.

Oxidative stress refers to an imbalance between oxidants and antioxidants. Although the relationship between oxidative stress and EndMT in SSc remains controversial [39], it has been validated that oxidative stress plays a pivotal role in the induction and progression of SSc [18]. Oxidants may directly activate fibroblasts or contribute to the maintenance of fibrosis by altering the balance between protease and anti-protease activity [40]. Inflammation is another important player in SSc. IL-6 and TNF-a levels have been demonstrated to be increased in the serum and skin of patients with SSc [41, 42]. Moreover, blocking IL-6 decreased collagen production in the BLM model of lung fibrosis [43]. Linagliptin has also been reported to possess anti-inflammatory and antioxidant properties [44, 45]. In our study, BLM-induced oxidative stress and inflammation were inhibited by linagliptin in vivo, indicating that the therapeutic effect of linagliptin against BLM-induced pulmonary fibrosis may also be attributed to its antiinflammatory and antioxidant properties.

TGF- $\beta$ mediated Smad-dependent and Smad-independent pathways, such as c-Abl kinase, protein kinase $c-\delta$, are most studied in EndMT. The Akt/mTOR pathway is an important smad-independent pathway involved in EndMT [32]. BLM induces EndMT in HUVECs via activation of this pathway [17]. Studies have shown that Salvia miltiorrhiza and Geniposide suppress BLM-induced EndMT by inhibiting the Akt/mTOR 
pathway $[16,22]$. Furthermore, this pathway may mediate oxidative stress, although further research is required [46]. Li et al have also reported that linagliptin inhibited high glucose-induced transdifferentiation of hypertrophic scar-derived fibroblasts to myofibroblasts via inactivation of the insulin-like growth factor (IGF)/Akt/mTOR pathway [47]. Here, we showed that BLM induced the activation of the Akt/mTOR pathway, and that linagliptin repressed this activation. Therefore, linagliptin may suppress EndMT by inhibiting the AKT/mTOR pathway in pulmonary fibrosis in the SSc model.

The study also has some limitations. First, the inhibitory effect of linaglitpin on EndMT in vitro was not repeated in human pulmonary endothelial cells in this study limited by objective conditions. Second, alternative models were not recruited to further validate these effects of linagliptin. These are issues that need to be addressed in future research.

\section{Conclusion}

We further confirmed the therapeutic effects of linagliptin on pulmonary fibrosis in SSc mouse model, which is based on its inhibitory effects on EndMT, oxidative stress and inflammation. Thus, linagliptin may be a promising candidate for the treatment of SSc-related pulmonary fibrosis.

\section{Declarations}

Funding This work was financially supported by the National Natural Science Foundation of China (9021087205).

Conflict of interest The authors confirm that they have no conflicts of interest to declare.

Data availability The datasets used and analyzed in the current study are available from the corresponding author on reasonable request.

Code availability Not applicable.

Authors' contributionsGengyun Sun conceived and guided the research. Biwei Pei performed the experiment, data analyses and wrote the manuscript. Na Zhang and Tingting Pang helped perform the analysis with constructive discussions. All the authors read, critically evaluated, gave their feedback, and edited the manuscript.

Ethical approval All animals were treated with humane conditions and institutional guidelines for the care and use of animals were followed.

Consent for publication Not applicable.

Consent to participate Not applicable.

\section{Acknowledgements}


This work was financially supported by the National Natural Science Foundation of China (9021087205).

\section{References}

1. Denton CP, Khanna D (2017) Systemic Sclerosis. Lancet 390:1685-1699

2. Elhai M, Meune C, Boubaya M et al (2017) Mapping and predicting mortality from systemic sclerosis. Ann Rheum Dis 76:1897-1905

3. Khanna D, Nagaraja V, Tseng C et al (2015) Predictors of lung function decline in scleroderma-related interstitial lung disease based on high-resolution computed tomography: implications for cohort enrichment in systemic sclerosis-associated interstitial lung disease trials. Arthritis Res Ther 17:372

4. Hu B, Phan SH (2013) Myofibroblasts. Curr Opin Rheumatol 25:71-77

5. Zeisberg EM, Tarnavski O, Zeisberg M et al (2007) Endothelial-to-mesenchymal transition contributes to cardiac fibrosis. Nat Med 13:952-961

6. Rieder F, Kessler SP, West GA et al (2011) Inflammation-induced endothelial-to-mesenchymal transition: a novel mechanism of intestinal fibrosis. Am J Pathol 179:2660-2673

7. Cruz-Solbes AS, Youker K (2017) Epithelial to Mesenchymal Transition (EMT) and Endothelial to Mesenchymal Transition (EndMT): Role and Implications in Kidney Fibrosis. Results Probl Cell Differ 60:345-372

8. AnbaraT, Sharifi M, Aboutaleb N (2020) Endothelial to Mesenchymal Transition in the Cardiogenesis and Cardiovascular Diseases. Curr Cardiol Rev 16:306-314

9. Mendoza FA, Piera-Velazquez S, Farber JL et al (2016) Endothelial cells expressing endothelial and mesenchymal cell gene products in Systemic Sclerosis-associated interstitial lung disease lung tissues. Arthritis Rheumatol 68:210-217

10. Klemann C, Wagner L, Stephan S, Hörsten SV (2016) Cut to the chase: a review of CD26/dipeptidyl peptidase-4's (DPP4) entanglement in the immune system. Clin Exp Immunol 185:1-21

11. Trzaskalski NA, Fadzeyeva E, Mulvihill EE (2020) Dipeptidyl Peptidase-4 at the Interface Between Inflammation and Metabolism. Clin Med Insights Endocrinol Diabetes 13:1179551420912972

12. Deacon CF (2020) Dipeptidyl peptidase 4 inhibitors in the treatment of type 2 diabetes mellitus. Nat Rev Endocrinol 16:642-653

13. Soare A, Györfi HA, Matei AE et al (2020) Dipeptidyl-peptidase-4 as a marker of activated fibroblasts and a potential target for the treatment of fibrosis in Systemic Sclerosis. Arthritis Rheumatol 72:137149

14. Xin Y, Wang X, Zhu M et al (2017) Expansion of CD26 positive fibroblast population promotes keloid progression. Exp Cell Res 356:104-113

15. Liu Y, Qi YC (2020) Vildagliptin, a CD26/DPP4 inhibitor, ameliorates bleomycin-induced pulmonary fibrosis via regulating the extracellular matrix. Int Immunopharmacol 87:106774

16. Qi Q, Mao Y, Tian Y et al (2017) Geniposide inhibited endothelial-mesenchymal transition via the mTOR signaling pathway in a bleomycin-induced scleroderma mouse model. Am J Transl Res 
9:1025-1036

17. Zhang W, Chen G, Ren J, Zhao Y (2013) Bleomycin induces endothelial mesenchymal transition through activation of mTOR pathway: a possible mechanism contributing to the sclerotherapy of venous malformations. $\mathrm{Br} \mathrm{J}$ Pharmacol 170:1210-1220

18. Grygiel-Górniak B, Puszczewicz M (2014) Oxidative damage and antioxidative therapy in systemic sclerosis. Mediators Inflamm. 2014:389582

19. Jomova K, Valko M (2011) Advances in metal-induced oxidative stress and human disease. Toxicology 283:65-87

20. Tang H, Mao J, Ye X et al (2020) SHIP-1, a target of miR-155, regulates endothelial cell responses in lung fibrosis. FASEB J 34:2011-2023

21. Chao J, Wang X, Zhang $Y$ et al (2016) Role of MCPIP1 in the EndothelialMesenchymal Transition Induced by Silica. Cell Physiol Biochem 40:309-325

22. Jiang Y, Hu F, Li Q, Shen C, Yang J, Li M (2019) Tanshinone IIA ameliorates the bleomycin-induced endothelial-tomesenchymal transition via the Akt/mTOR/p70S6K pathway in a murine model of systemic sclerosis. Int Immunopharmacol 77:105968

23. Yoshizaki A, Iwata Y, Komura K et al (2008) CD19 Regulates Skin and Lung Fibrosis via Toll-Like Receptor Signaling in a Model of Bleomycin-Induced Scleroderma. Am J Pathol 172:1650-1663

24. Harrison JH, Lazo JS (1987) High dose continuous infusion of bleomycin in mice: A new model for drug-induced pulmonary fibrosis. J Pharmacol Exp Ther 24:1185-1194

25. Bhattacharyya S, Wang W, Morales-Nebreda $L$ et al (2016) Tenascin-C drives persistence of organ fibrosis. Nat Commun 7:11703

26. Bhattacharyya S, Wang W, Tamaki Z et al (2018) Pharmacological Inhibition of Toll-Like Receptor-4 Signaling by TAK242 Prevents and Induces Regression of Experimental Organ Fibrosis. Front Immunol 9:2434

27. Leach HG, Chrobak I, Han R, Trojanowska M (2013) Endothelial cells recruit macrophages and contribute to a fibrotic milieu in Bleomycin lung injury. Am J Respir Cell Mol Biol 49:1093-1101

28. Caam A, Vonk M, Hoogen F, Lent P, Kraan P (2018) Unraveling SSc pathophysiology; the myofibroblast. Front Immunol 9:2452

29. Postlethwaite AE, Shigemitsu H, Kanangat S (2004) Cellular origins of fibroblasts: Possible implications for organ fibrosis in systemic sclerosis. Curr Opin Rheumatol 16:733-738

30. Herzog EL, Bucala R (2010) Fibrocytes in health and disease. Exp Hematol 38:548-556

31. Lamouille S, Xu J, Derynck R (2014) Molecular mechanisms of epithelial-mesenchymal transition. Nat Rev Mol Cell Biol 15:178-196

32. Piera-Velazquez S, Jimenez SA (2019) Endothelial to Mesenchymal Transition: Role in Physiology and in the Pathogenesis of Human Diseases. Physiol Rev 99:1281-1324

33. Ota Y, Kuwana M (2020) Endothelial cells and endothelial progenitor cells in the pathogenesis of systemic sclerosis. Eur J Rheumatol 7:S139-S146 
34. Suzuki T, Tada Y, Gladson S et al (2017) Vildagliptin ameliorates pulmonary fibrosis in lipopolysaccharide-induced lung injury by inhibiting endothelial-to-mesenchymal transition. Respir Res 18:177

35. Hashimoto N, Phan SH, Imaizumi K et al (2010) Endothelial-Mesenchymal Transition in BleomycinInduced Pulmonary Fibrosis. Am J Respir Cell Mol Biol 43:161-172

36. Kokudo T, Suzuki Y, Yoshimatsu Y, Yamazaki T, Watabe T, Miyazono K (2008) Snail is required for TGFbeta-induced endothelial-mesenchymal transition of embryonic stem cell-derived endothelial cells. J Cell Sci 121:3317-3324

37. Mammoto T, Muyleart M, Konduri GG, Mammoto A (2018) Twist1 in Hypoxia-induced Pulmonary Hypertension through Transforming Growth Factor- $\beta$-Smad Signaling. Am J Respir Cell Mol Biol 58:194-207

38. Shi S, Srivastava SP, Kanasaki M et al (2015) Interactions of DPP-4 and integrin $\beta 1$ influences endothelial-to-mesenchymal transition. Kidney Int 88:479-489

39. Thuan DTB, Zayed H, Eid AH et al (2018) A Potential Link Between Oxidative Stress and Endothelialto-Mesenchymal Transition in Systemic Sclerosis. Front Immunol 9:1985

40. Svegliati S, Spadoni T, Moroncini G, Gabrielli A (2018) NADPH oxidase, oxidative stress and fibrosis in Systemic Sclerosis. Free Radic Biol Med 125:90-97

41. Khan K, Xu S, Nihtyanova S, Derrett-Smith E et al (2012) Clinical and pathological significance of interleukin 6 overexpression in systemic sclerosis. Ann Rheum Dis 71:1235-1242

42. Pehlivan Y, Onat AM, Ceylan N et al (2012) Serum leptin, resistin and TNF-a levels in patients with systemic sclerosis: the role of adipokines in scleroderma. Int J Rheum Dis 15:374-379

43. Saito F, Tasaka S, Inoue K et al (2008) Role of interleukin-6 in bleomycin-induced lung inflammatory changes in mice. Am J Respir Cell Mol Biol 38:566-571

44. Birnbaum Y, Tran D, Bajaj M, Ye Y (2019) DPP-4 inhibition by linagliptin prevents cardiac dysfunction and inflammation by targeting the NIrp3/ASC inflammasome. Basic Res Cardiol 114:35

45. Sonoda MN, Inoue T, Kimura S et al (2020) The dipeptidyl peptidase-4 inhibitor, linagliptin, improves cognitive impairment in streptozotocin-induced diabetic mice by inhibiting oxidative stress and microglial activation. PLoS One 15:e0228750

46. Jiang F, Liu G, Dusting GJ, Chan EC (2014) NADPH oxidase-dependent redox signaling in TGF- $\beta$ mediated fibrotic responses. Redox Biol 15:267-272

47. Li Y, Zhang L, Zhou Q et al (2019) Linagliptin inhibits high glucose-induced trans-differentiation of hypertrophic scar derived fibroblasts to myofibroblasts via IGF/Akt/mTOR signaling pathway. Exp Dermatol 28:19-27

\section{Figures}




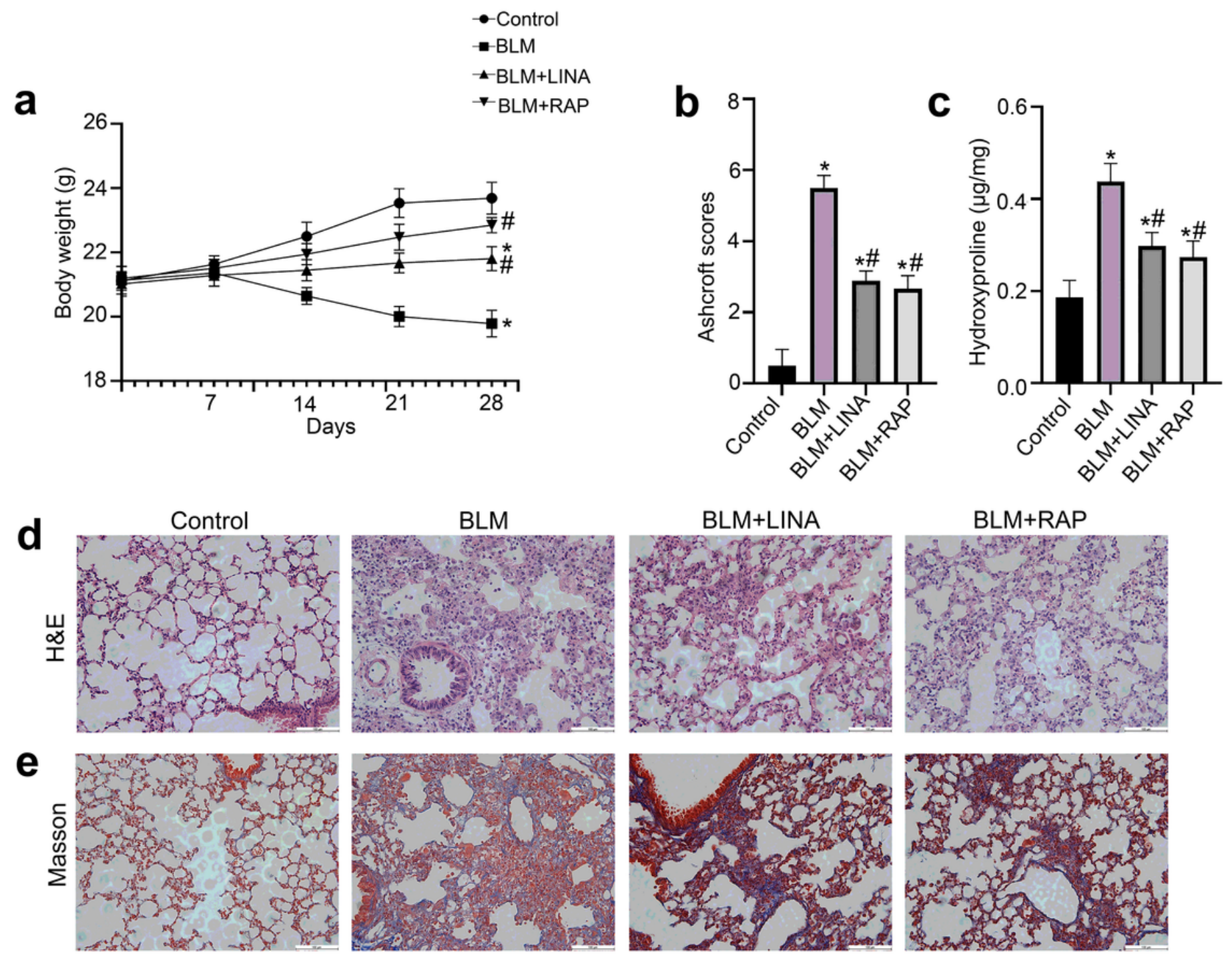

Figure 1

Linagliptin (LINA) and Rapamycin (RAP) ameliorated pulmonary fibrosis in the BLM-induced SSc mouse model. Mouse model was induced by subcutaneously injection of BLM, and all the mice were sacrificed on day 28. (a) Mice body weight were measured. (b) Ashcroft scores were calculated based on H\&E staining. (c) The hydroxyproline content in mice lung tissues were determined. (d) Representative images of H\&E staining (200x) and (e) Masson's trichrome staining (200x) of paraffin lung sections. The results are presented as means $\pm S D(n=6) . * P<0.05$ vs control; $\# P<0.05$ vs $B L M$ 

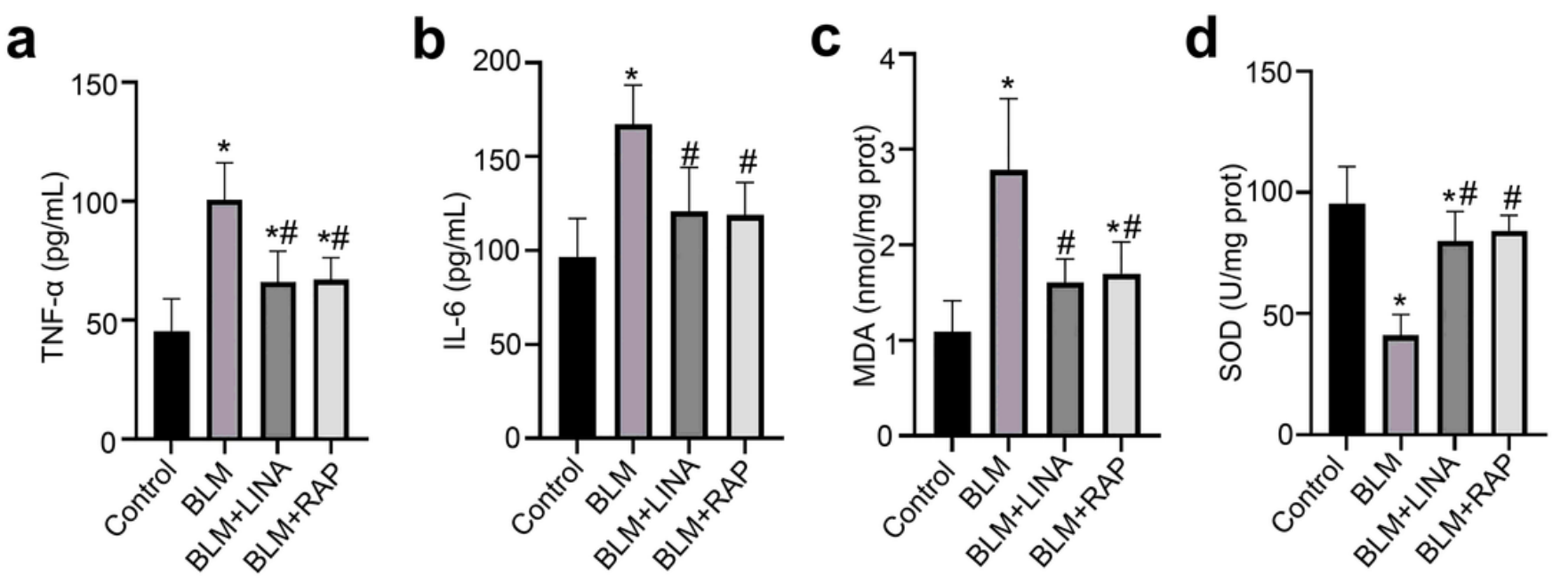

Figure 2

LINA and RAP mitigated BLM-induced inflammation and oxidative stress. BALF and lungs were harvested on day 28. The levels of TNF- $a(a)$ and IL-6 (b) in the BALF in different groups were measured by ELISA. The MDA content (c) and SOD activity (d) in lungs in different groups were measured by respective kits. The results are presented as means $\pm S D(n=6)$. ${ }^{*}<<0.05$ vs control; \#P $<0.05$ vs BLM 
a
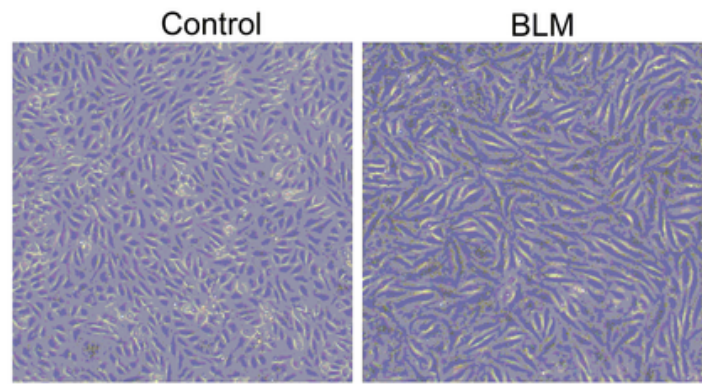

BLM+LINA

BLM+RAP
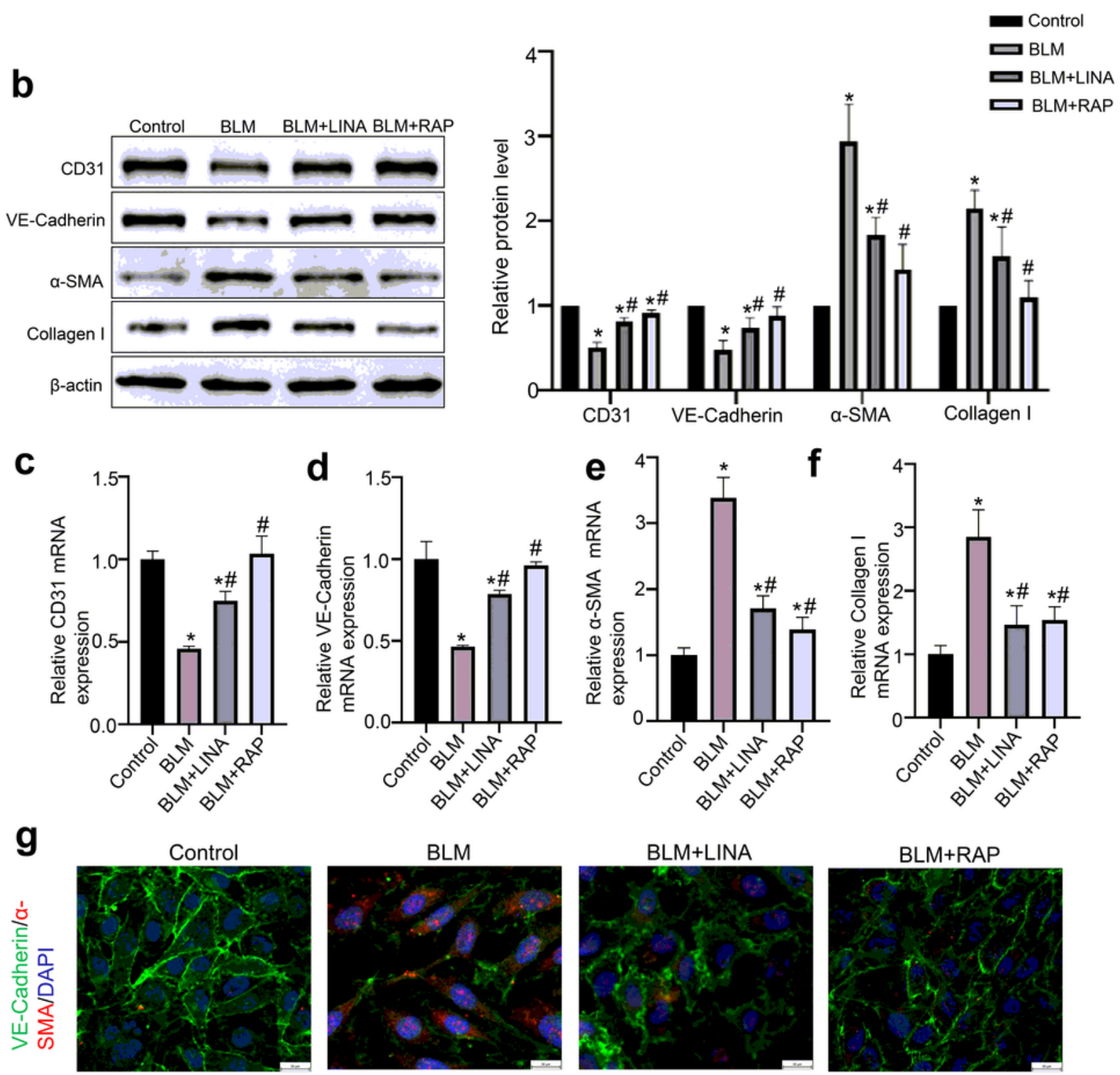

\section{Figure 3}

LINA and RAP attenuated BLM-induced EndMT in vitro. (a) BLM treatment for $48 \mathrm{~h}$ induced morphological changes of HUVECs, while addition of LINA or RAP suppressed these changes (100x). (b) Changes of protein and $(c, d, e, f)$ mRNA levels of EndMT-related markers were measured by western blotting and RT-qPCR. (g) Immunofluorescence double staining showed the expression change and location of VE-Cadherin and a-SMA in HUVECs in different groups. Nuclei were stained by DAPI. The 
results are presented as means $\pm S D$ from three independent experiments. ${ }^{*} \mathrm{P}<0.05$ vs control; $\# \mathrm{P}<0.05$ vs BLM

a
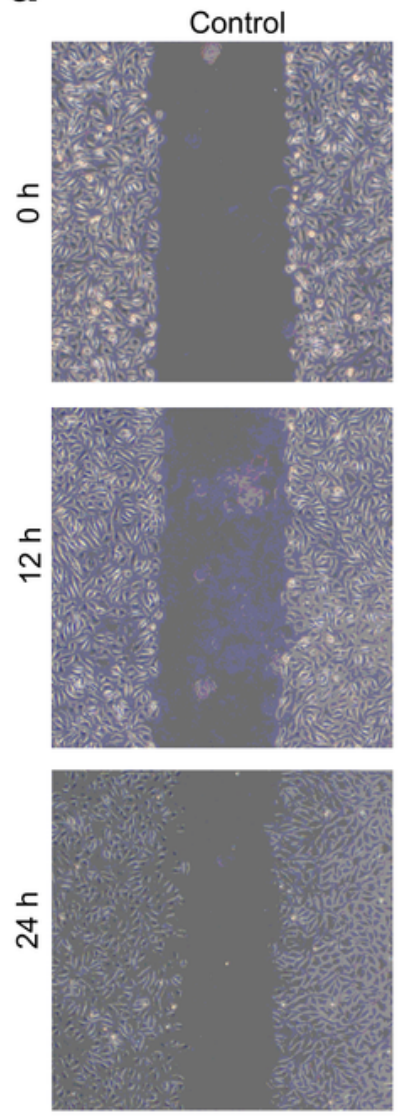

b
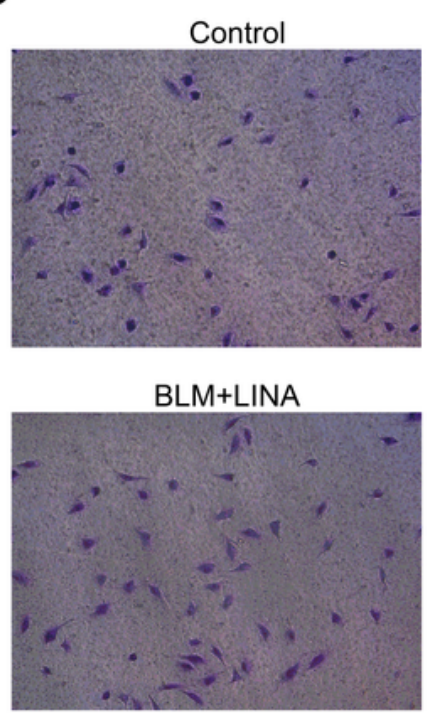

BLM
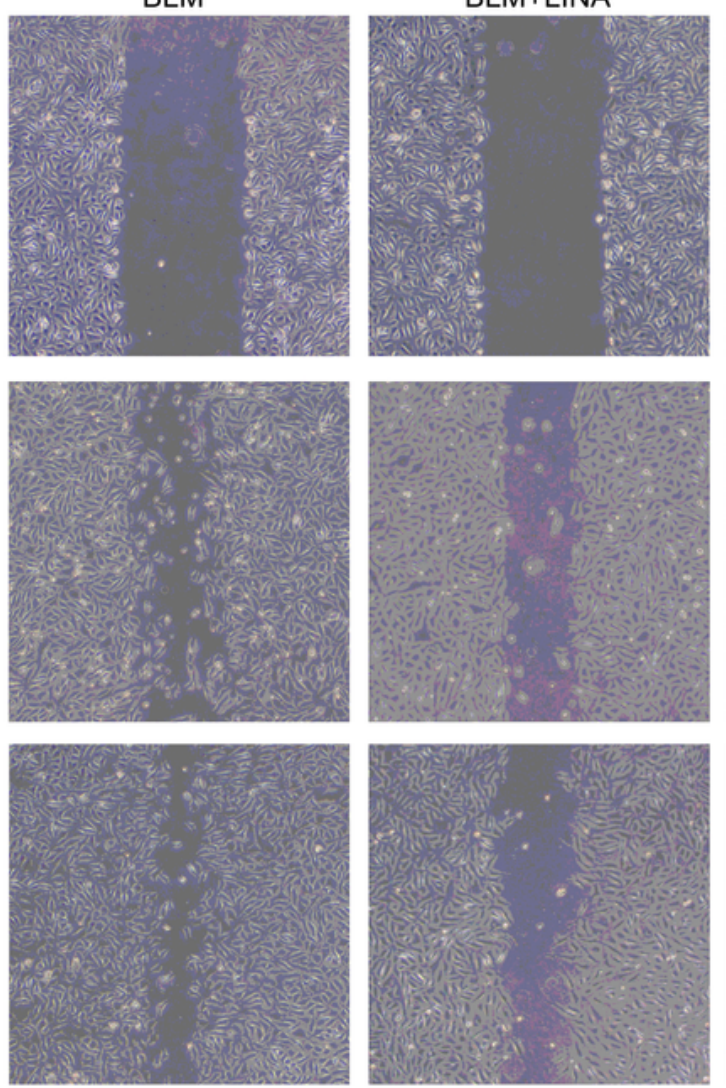

$B L M+R A P$
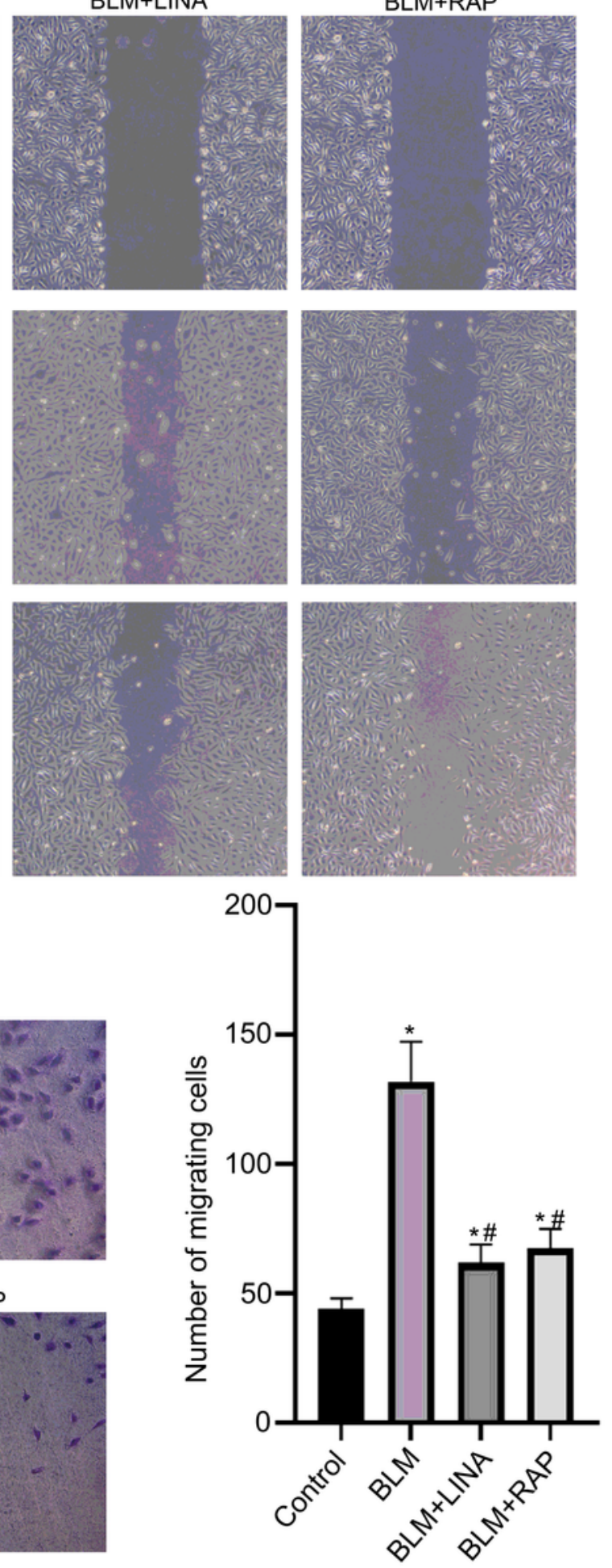

Figure 4

LINA and RAP inhibited BLM-induced enhanced migration ability. The inhibitory effects of LINA and RAP

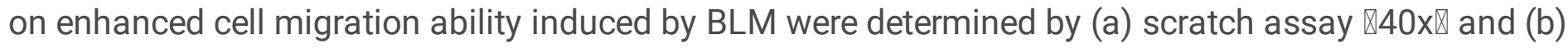


transwell migration assay $₫ 200 x \rrbracket$. The results are presented as means $\pm S D$ from three independent experiments. ${ }^{*} \mathrm{P}<0.05$ vs control; $\# \mathrm{P}<0.05$ vs $\mathrm{BLM}$

a
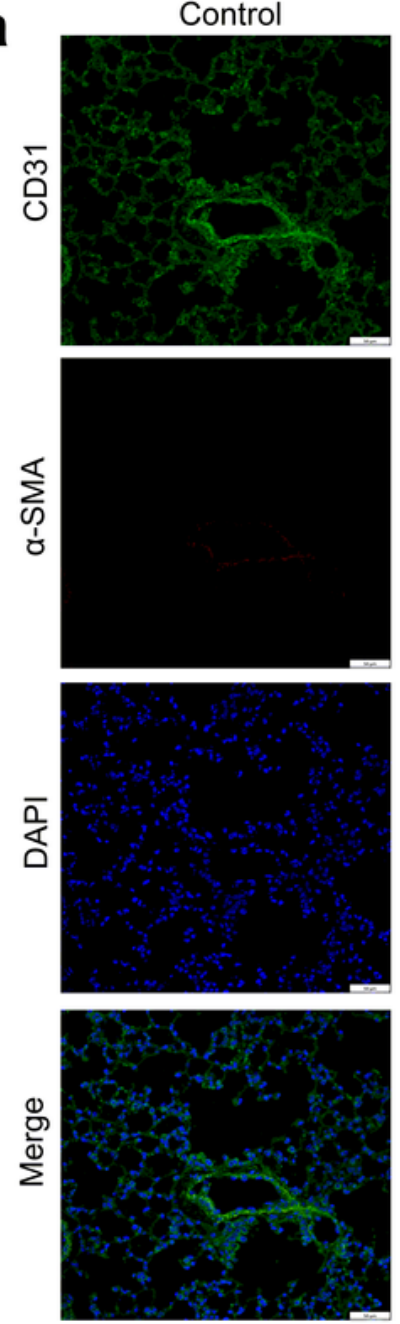

b

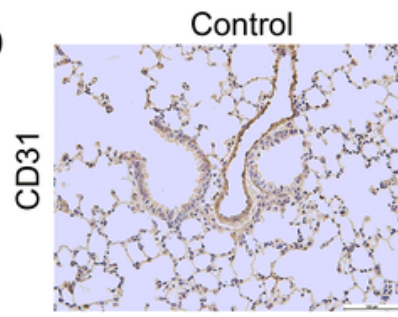

c

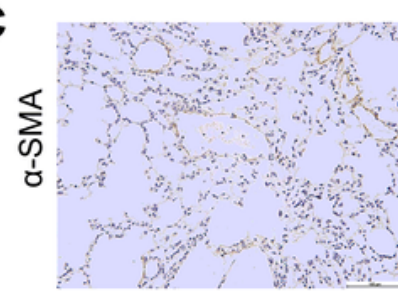

BLM
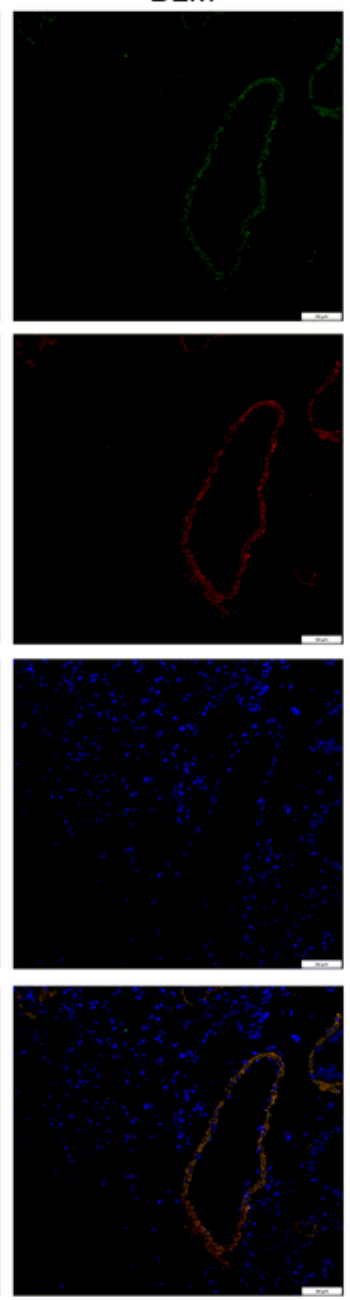

BLM
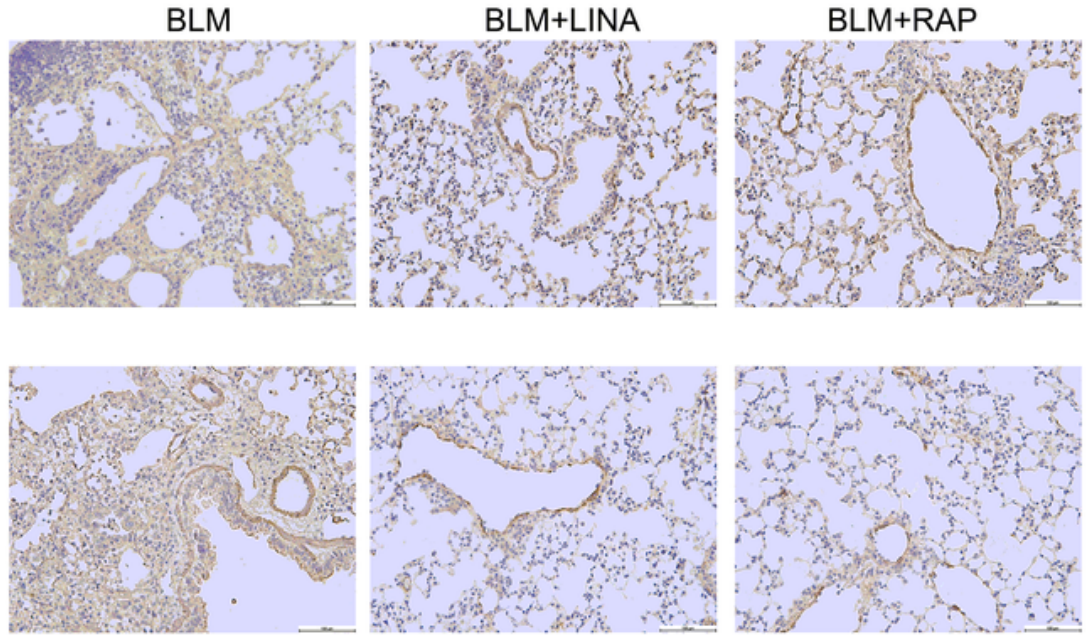
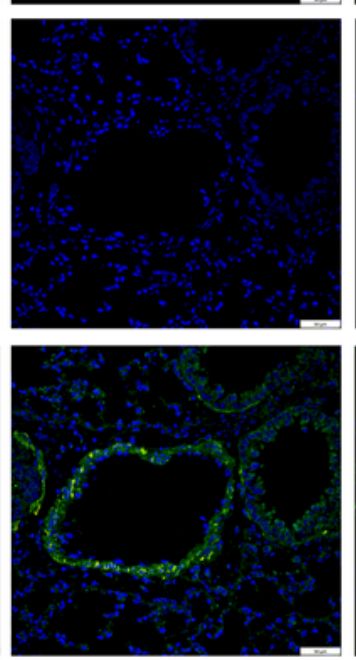

BLM+LINA

BLM+LINA
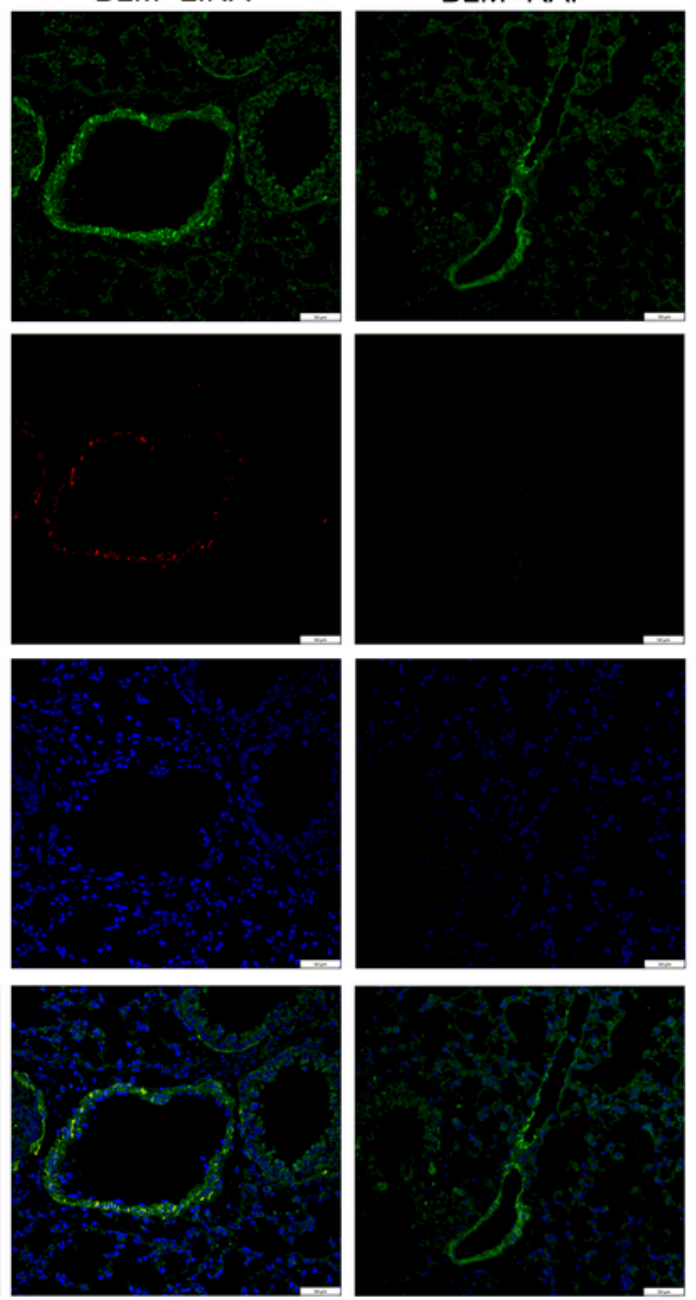

$\mathrm{BLM}+\mathrm{RAP}$

BLM+RAP

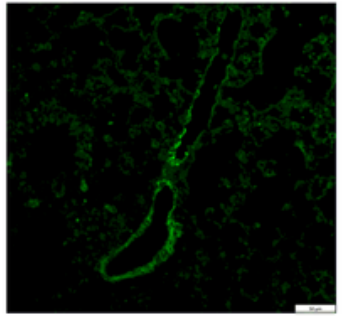

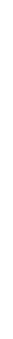
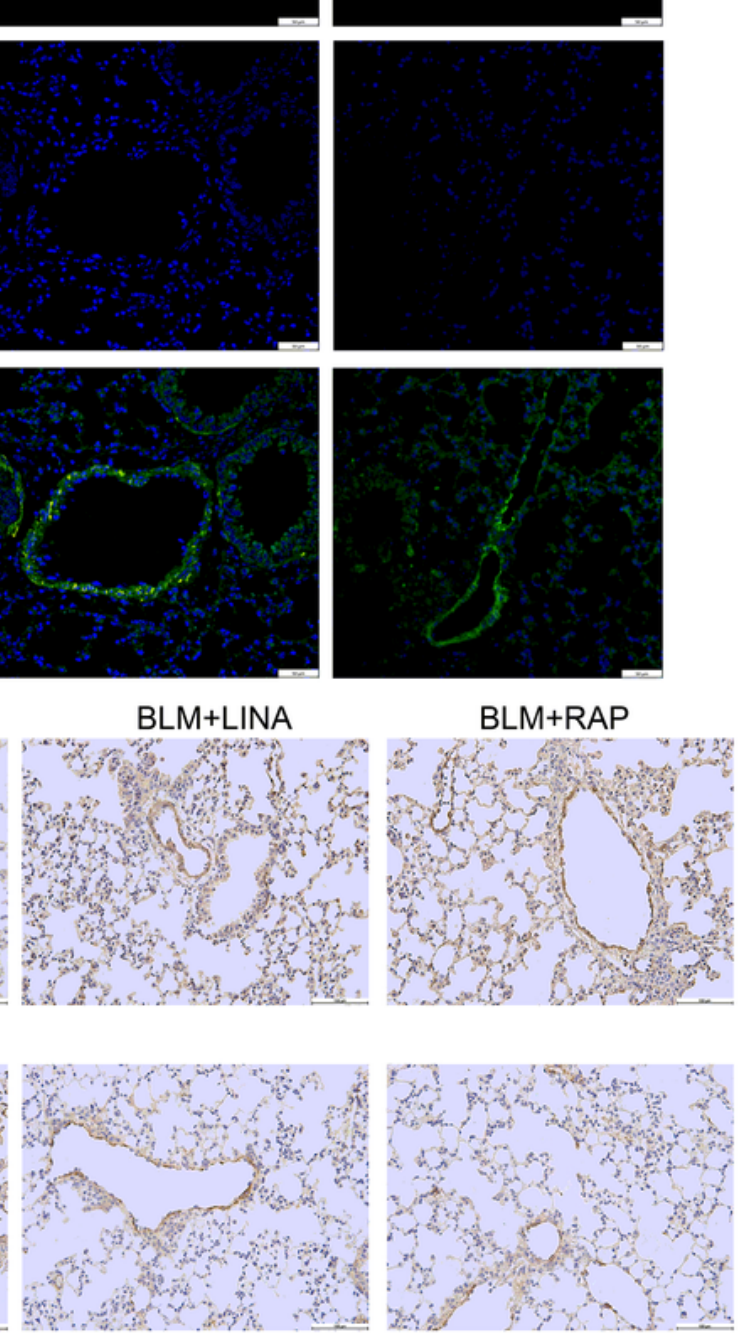

Figure 5

LINA and RAP attenuated the EndMT in vivo. (a) Immunofluorescence double staining showed the expression change and location of CD31 and a-SMA in lungs in different groups. Nuclei were stained by 
DAPI. (b) Immunohistochemistry analysis of CD31 (200x) and (c) a-SMA (200x) expressed at the intima of the capillary vessels in lung tissues in different groups. * $\mathrm{P}<0.05$ vs control; \#P $<0.05$ vs BLM

a

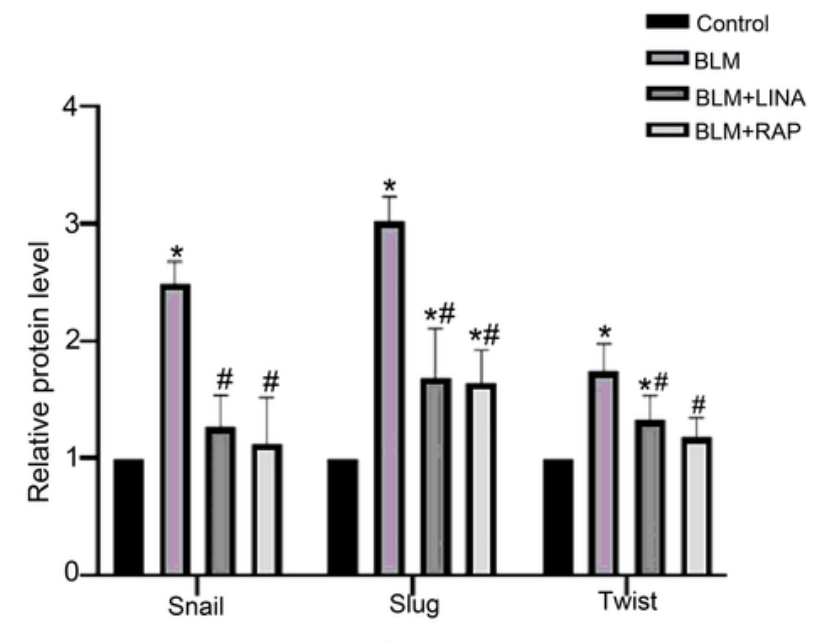

b

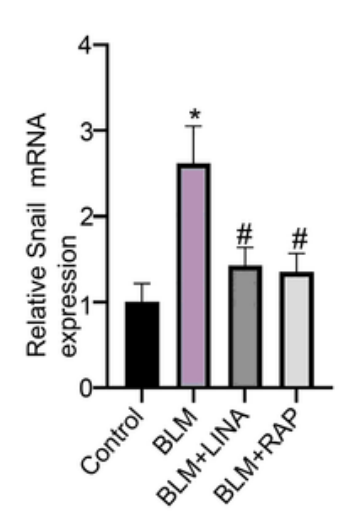

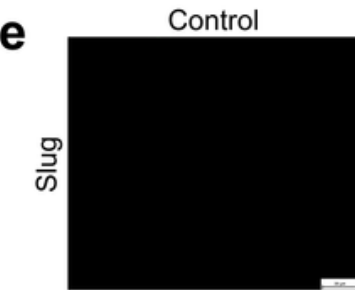
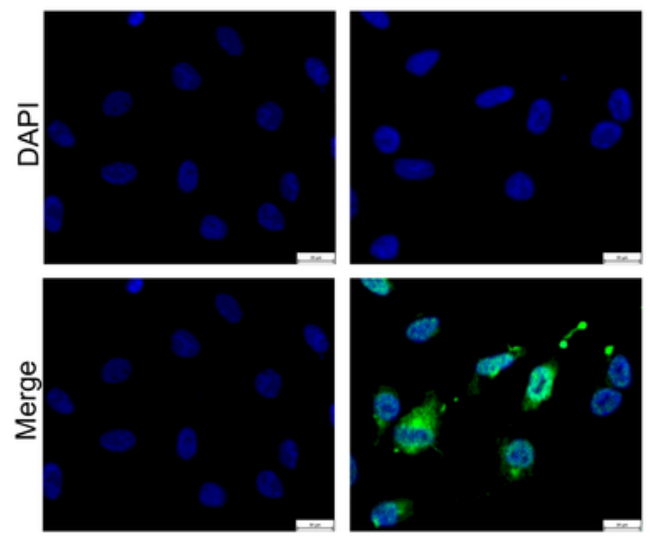

C

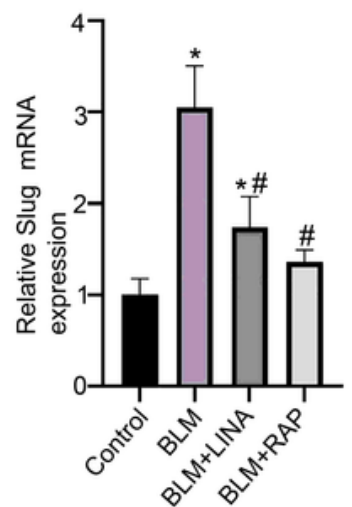

BLM

BLM+LINA
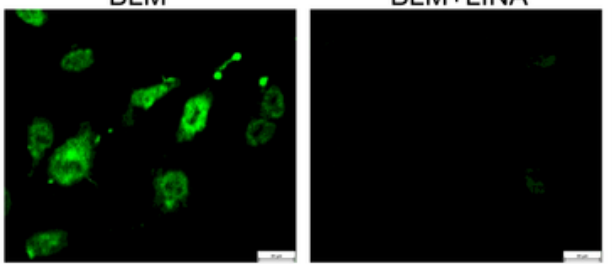

d

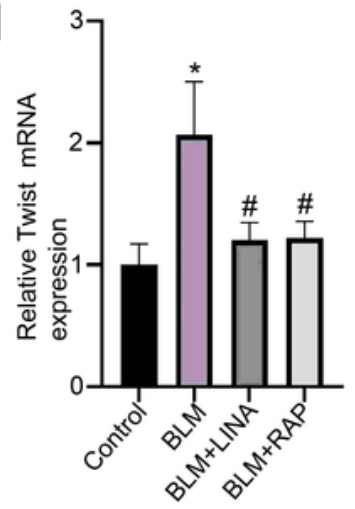

BLM+RAP

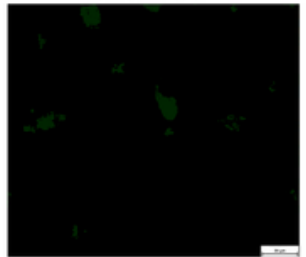

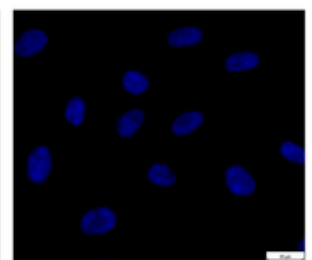
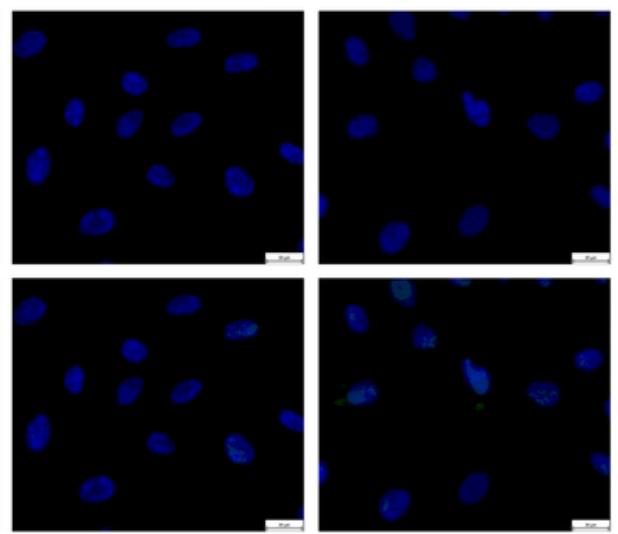

\section{Figure 6}

LINA and RAP inhibited EndMT-related transcription factors in vitro. (a) Protein and (b, c, d) mRNA levels of Snail, Slug, Twist in HUVECs were determined by western blotting and RT-qPCR. (e) Nuclear location of 
Slug was detected by immunofluorescence analysis. The results are presented as means $\pm S D$ from three independent experiments. ${ }^{*} \mathrm{P}<0.05$ vs control; \#P $<0.05$ vs BLM

a

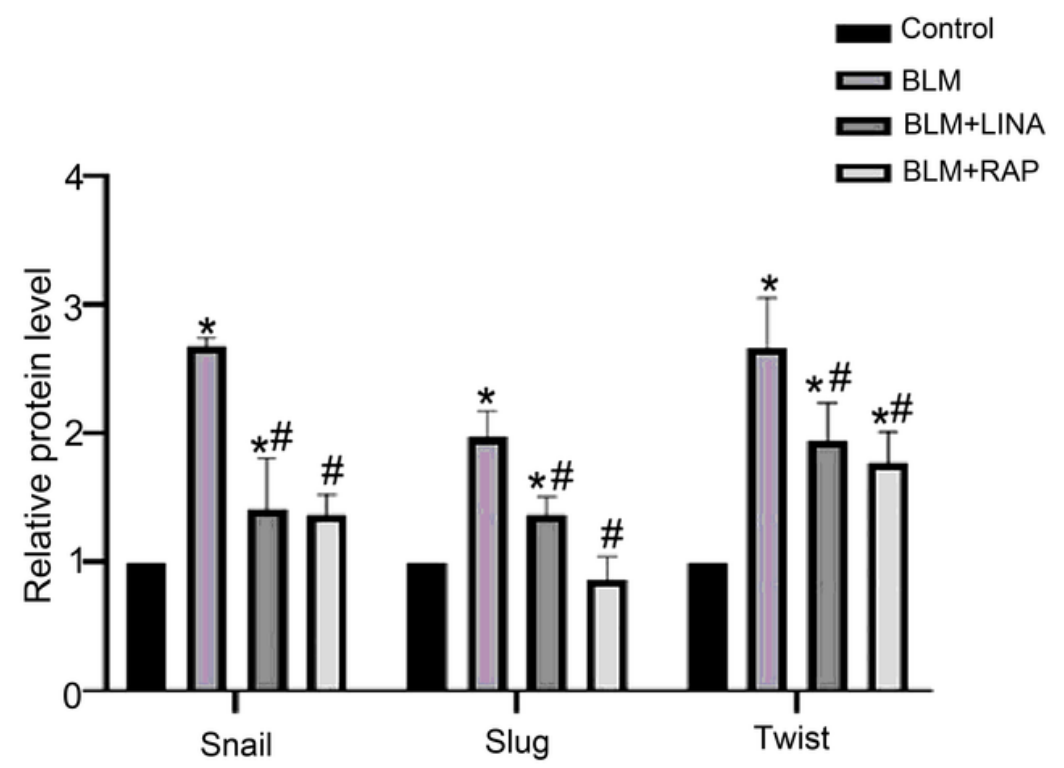

b
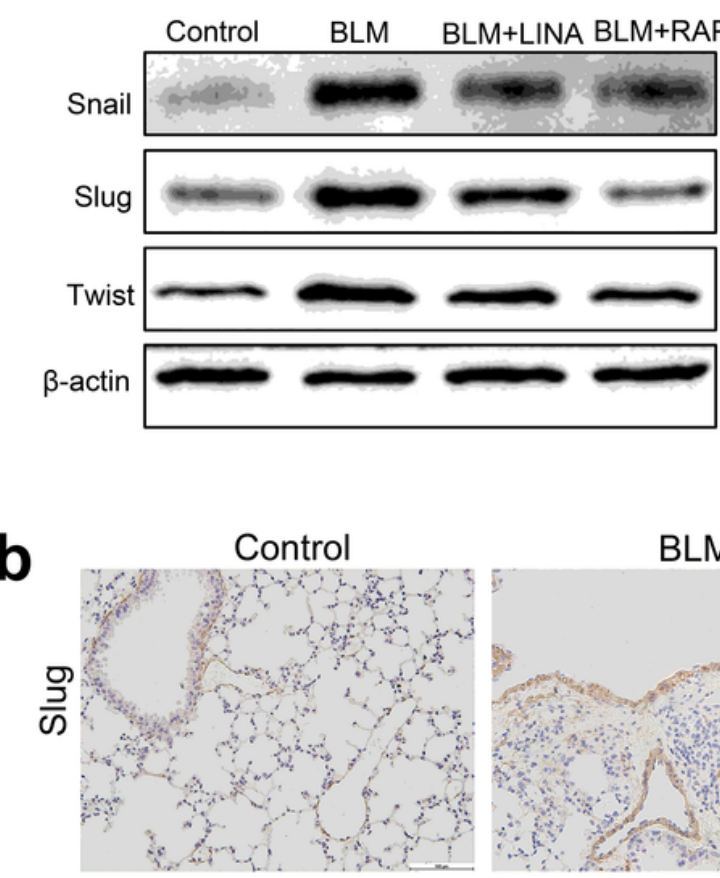

BLM

BLM+LINA

BLM+RAP
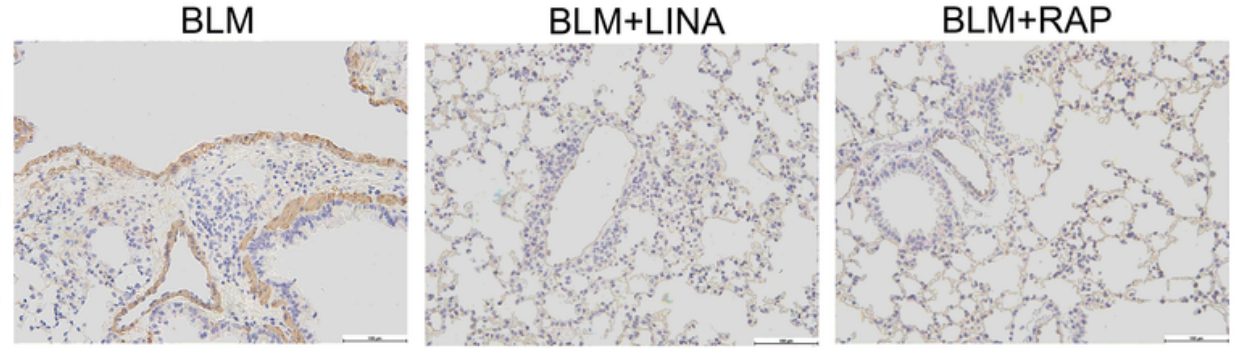

Figure 7

LINA and RAP inhibited EndMT-related transcription factors in vivo. (a) Protein levels of Snail, Slug, Twist in lung tissues were determined by western blotting. (b) Immunohistochemistry analysis of Slug (200x) expressed at the intima of the capillary vessels in lung tissues in different groups. The results are presented as means $\pm S D(n=6)$. ${ }^{*}<0.05$ vs control; $\# P<0.05$ vs $B L M$

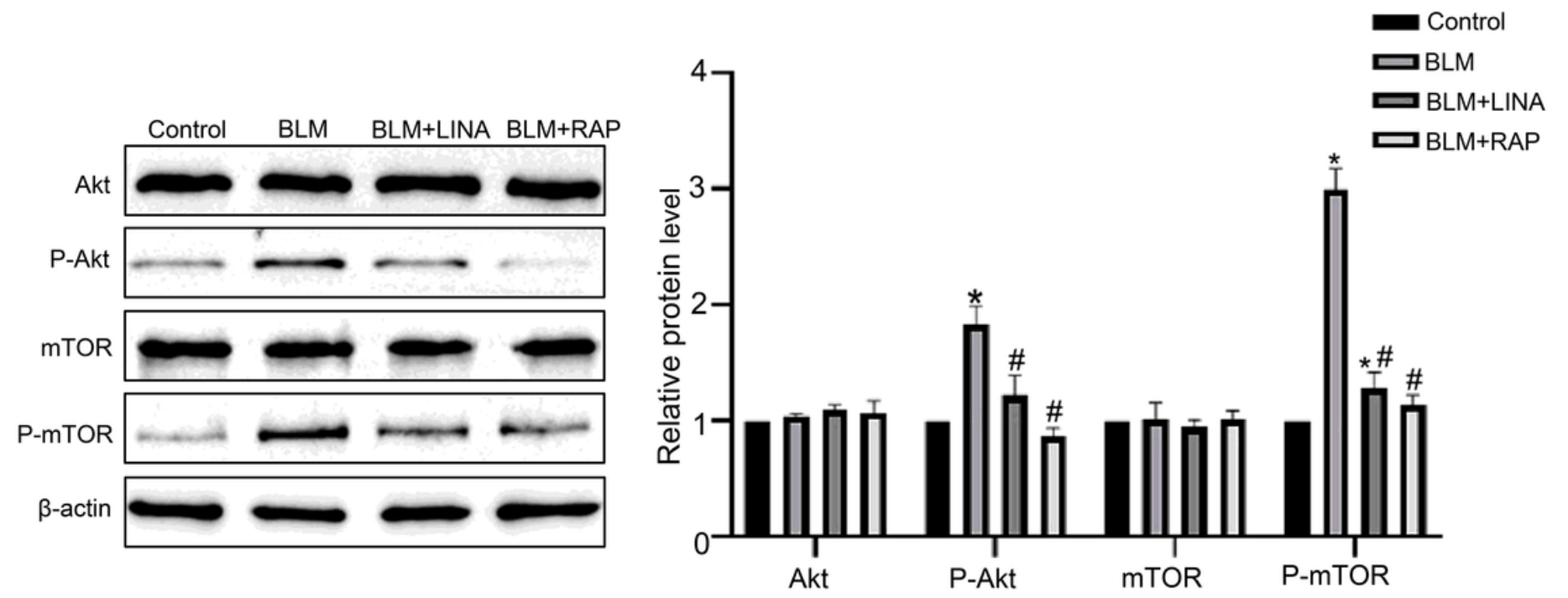




\section{Figure 8}

LINA and RAP inhibited the Akt/mTOR pathway in vivo. Protein levels of Akt, P-Akt, mTOR, P-mTOR were determined by western blotting. The results are presented as means $\pm S D(n=6) .{ }^{*} P<0.05$ vs control; \#P $<0.05$ vs BLM 